\title{
On Model Structure Selection for Nonparametric Prediction Methods
}

\author{
Anders Stenman \\ Department of Electrical Engineering \\ Linköpings universitet, SE-581 83 Linköping, SWEDEN \\ URL: http://www.control.isy.liu.se \\ Email: stenmaneisy.liu.se
}

March 16, 2000

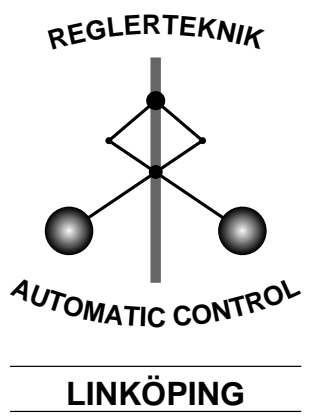

Report no.: LiTH-ISY-R-2231

To be presented at the IFAC Symposium on System Identification Santa Barbara, California, USA, June 21-23, 2000.

Technical reports from the Automatic Control group in Linköping are available by anonymous ftp at the address ftp.control.isy.liu.se. This report is contained in the PDF file 2231.pdf. 


\title{
ON MODEL STRUCTURE SELECTION FOR NONPARAMETRIC PREDICTION METHODS
}

\author{
Anders Stenman $*, 1$ \\ * Department of Electrical Engineering, Linköpings universitet, \\ SE-581 83 Linköping, SWEDEN. Fax: +4613282622
}

\begin{abstract}
In this paper we continue to explore identification of nonlinear systems using the previously proposed concept of model-on-demand. The idea is to estimate the process dynamics locally and on-line using process data stored in a database, and has in earlier contributions proven to be capable to produce results comparable to (or better than) other nonlinear blackbox approaches. The modeling part of the method is based on local polynomial modeling ideas. This has several implications on the choice of model structure, which is discussed at length in the paper. It is concluded that the NARX structure should be considered as the default choice in the local polynomial context. Furthermore, it is shown that the predictions in some situations can be enhanced by tuning other parameters that are special for the nonparametric case. The usefulness of the method is illustrated in numerical simulations. For the chosen application it is shown that the prediction errors are in order of magnitude directly comparable to more established modeling tools such as artificial neural nets.
\end{abstract}

Keywords: system identification, local polynomial modeling

\section{INTRODUCTION}

The problem of modeling dynamical systems from experimental data with poor a priori knowledge of the system is usually referred to as black-box modeling (Ljung, 1999). It has traditionally been solved using linear models of different sophistication. However, problems usually occur when encountering highly nonlinear systems that poorly allow themselves to be approximated by linear models. As a consequence of this, the interest for nonlinear modeling alternatives like neural networks and radial basis functions has been growing in recent years (Sjöberg et al., 1995; Chen et al., 1990). When the number of observations grows very large, however, this approach appears less attractive because of the difficulties in specifying model structure and the complexity of the associated optimization problem. Inspired by ideas from local modeling and database systems technology, we have taken a conceptually different point of view: We assume that all available data are stored in a database,

\footnotetext{
1 Email: stenman@isy.liu.se
}

and that local models are built "on demand" as the actual need arises.

It is clear that many possibilities for constructing the local model exist. In this contribution we will focus on different nonparametric methods derived in earlier contributions (Stenman et al., 1996; Stenman, 1999), and will address some of the design considerations that have to be taken into account when using such methods for prediction and simulation.

The organization of the paper is as follows: Section 2 states the basic problem considered, whereby Section 3 shows how it can be solved using the modelon-demand concept. The different aspects of model structure selection associated with the method are discussed in Section 4, and are exemplified in Section 5. In Section 6, finally, the conclusions are drawn.

\section{PRELIMINARIES}

The class of systems considered here will be nonlinear, multi-input, single-output (MISO) systems in 
discrete time, governed in input-output form by the dynamic relationship

$$
y(t)=m(\varphi(t))+v(t)
$$

where

$$
\varphi(t)=\varphi\left(Z^{t-1}\right)
$$

is a regression vector constructed from past measurements

$$
Z^{N} \triangleq\{(u(1), y(1)), \ldots,(u(N), y(N))\} .
$$

The goal of the modeling is to produce a good predictor, i.e., one that estimates

$$
\hat{y}(t)=\mathrm{E}(y(t) \mid \varphi(t))
$$

as well as possible, utilizing all the available information contained in $\varphi(t)$. This problem has traditionally been solved by parametric black-box approaches. In this contribution we shall instead utilize the recently proposed concept of model-on-demand for prediction and simulation purposes.

\section{MODEL-ON-DEMAND}

The basic principles behind the model-on-demand philosophy is as follows: Given a database of observed output-regressor pairs from the plant $\{(y(k), \varphi(k))\}$, a local prediction is formed by retrieving relevant data from the database and applying a suitable modeling operation.

For the modeling part we have adopted a "sliding window" approach. That is, for each operating point $\varphi(t)$, a local model is identified via the weighted regression problem; minimize

$$
\sum_{k=1}^{N} \ell(y(k)-m(\varphi(k), \beta)) \cdot w_{k}\left(\varphi_{t}\right),
$$

subject to $\beta$. Here $w_{k}(\cdot)$ are weights that control the locality and $\ell(\cdot)$ is a scalar-valued and positive norm function.

The weights can of course be selected in many ways. In the statistics they are often implicitly selected according to

$$
w_{k}(\varphi(t))=W\left(\frac{\|\varphi(k)-\varphi(t)\|_{\mathcal{M}}}{h}\right),
$$

where $h$ is a bandwidth parameter controlling the size of the local neighborhood, $\|u\|_{\mathcal{M}}$ is a scaled distance function on the regressor space, and $W(\cdot)$ is a window function (usually referred to as the kernel) assigning weights to each remote data point according to its distance from $\varphi(t)$. The kernel is typically a bellshaped function with support on the interval $[0,1]$, see Figure 1, where some commonly used windows are depicted. In Stenman (1999) we also studied a direct optimization scheme that explicitly determines the optimal weights. It has been shown that this might result in better accuracy in some cases (Stenman et al., 1997).

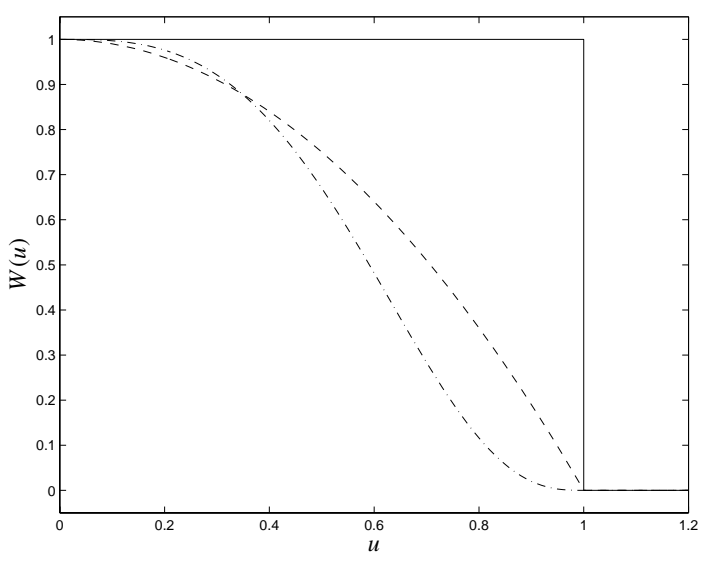

Fig. 1. Some commonly used window functions: Uniform (solid), Epanechnikov, $W(u)=\left(1-u^{2}\right)_{+}$ (dashed), and tricube, $W(u)=\left(1-u^{3}\right)_{+}^{3}$ (dashdotted).

\subsection{Local Model Structures}

In principle, it is possible to use any model structure as local model in (2). However, if a quadratic norm, $\ell(\varepsilon)=\frac{1}{2} \varepsilon^{2}$, is used and the model is linear in the parameters, the estimate can be explicitly computed using ordinary weighted least squares. This idea of estimating $\beta$ has a long tradition within the statistics and is usually referred to as local polynomial modeling (Fan and Gijbels, 1996).

For simplicity we assume a local linear model structure,

$$
m(\varphi(k), \beta)=\beta_{0}+\beta_{1}^{T}(\varphi(k)-\varphi(t))
$$

as the the default model in the sequel of the paper. Thus, if $\hat{\beta}_{0}$ and $\hat{\beta}_{1}$ denote the minimizers of (2) using the model (3), it is easy to realize that an estimate of $y(t)$ (i.e., the one-step-ahead prediction), is given by

$$
\hat{y}(t)=m(\varphi(t), \hat{\beta})=\hat{\beta}_{0} .
$$

Note that each optimization of (2) produces a single local prediction $\hat{y}(t)$ corresponding to the current regression vector $\varphi(t)$. To obtain predictions at other locations in the regressor space, the weights change and new optimization problems have to be solved. This is in contrast to the global modeling approach where the model is fitted to data only once. Since no global description is obtained, estimators like (2) is often called nonparametric.

\subsection{Model Tuning}

It is well known that the bandwidth $h$ has a critical impact on the resulting estimate, since it governs a tradeoff between the bias and variance errors of the estimate. Methods that use the available data to produce a good bandwidth are usually referred to as bandwidth selectors, and have been thoroughly studied within the statistical literature, see for instance Fan and Gijbels (1996) and the references therein. They can roughly be 
divided into "classical" methods which are based on cross-validation ideas, and "plug-in" methods which rely on minimizing asymptotic MSE expressions. The majority of the bandwidth selectors proposed so far, though, have been of global type, i.e., they produce a single global value. However, adaptive (i.e., local) methods, which select local bandwidths on-line for each estimation point, have gained a significant interest in recent years, even though the development of them still seems to be an open and active research area. See Stenman (1999) for a detailed discussion.

The scaling matrix $\mathcal{M}$ that controls the distance function can be optimized in a similar way. The simplest choice, though, is to let it be the identity matrix. This corresponds to the usual Euclidean norm. A diagonal scaling matrix transforms the radially symmetric distance function into an axis parallel ellipse. By also allowing cross-terms in $\mathcal{M}$ it is possible to make the ellipse arbritrary oriented. Selecting the scaling matrix is very important, especially when the regressor components have very different magnitudes. An obvious default choice, which has been adopted here, is to make it proportional to the inverse covariance of the regressors.

\subsection{Extensions}

It is clear that several extensions of the local polynomial regression idea exist. One possibility is to replace (2) with a localized maximum likelihood criterion (Tibshirani and Hastie, 1987), although this will increase the computational burden even more. Recent work by Kulhavý and Ivanova (1999) has also shown how it is possible to incorporate a priori information to the local model through a localized Bayesian estimation approach.

\section{DESIGN CONSIDERATIONS}

In order to obtain a local model that is suitable for prediction or simulation, there are a number of design questions which have to be taken into consideration:

(1) Which quantities, constructed from past measurements, should be included in the regression vector $\varphi(t)$ ?

(2) Which model structure should be used?

(3) Which criteria should be used when determining which data that are relevant for the local fit?

The first question is the same for all modeling approaches (linear as well as nonlinear ones) and in most situations it turns out that the most useful choices of regression vectors are those who are built up by past inputs and outputs. The second question appears in parametric black-box modeling too, but then usually includes a wide range of different parameterizations. In a local modeling framework, we essentially just have to decide upon the polynomial degree and the norm for the fit. The third question is rather unique for the nonparametric estimation problem, because of its local properties. Nevertheless, it is an important one, since we can emphasize different features in data with different relevance measures.

\subsection{Model Structures for Nonparametric Methods}

For parametric models, it is well known that adding an extra regressor component enables an increased flexibility, but that this comes to the price of an increased variability of the estimated parameters. It is thus often desirable to keep the dimension of the regression vector as low as possible. This conclusion still holds and is perhaps even more important in the nonparametric case. It is a well-known fact that the rate of convergence typically gets slower in higher regressor dimensions. This is primarily due to the socalled curse of dimensionality, which follows from the sparseness of data in the multidimensional regressor space. Another, but yet equally important reason is the dataset searching problem associated with the local modeling. Certain storage structures, like $k$ - $d$ trees (Bentley, 1975), have proven to be most efficient for a moderate number of dimensions.

As we shall see later, a possible alternative to restrict the size of the regression vector, is to impose restrictions on the mapping $m(\cdot)$. One such possibility is to assume that the regression function is global in certain regressor directions. This is usually referred to as conditionally parametric models, and could be a useful weapon to combat the curse-of-dimensionality.

Let us here review different choices on model structures and their ability to be adapted to the local polynomial framework. The discussion will be made assuming single-input, single-output systems. Generalization to the multiple-input, single-output case is straightforward however; think of $u(t)$ as a vector of inputs.

4.1.1. NFIR Structures The simplest nonlinear dynamical model is the nonlinear finite impulse response model, NFIR, which only uses past inputs as regressors, i.e.,

$y(t)=m\left(u\left(t-n_{k}\right), \ldots, u\left(t-n_{b}-n_{k}+1\right)\right)+e(t)$.

Here $n_{b}$ and $n_{k}$ denote the number of past inputs and the time delay, respectively. The corresponding predictor is obtained by deleting the noise term. That is,

$$
\hat{y}(t)=m\left(u\left(t-n_{k}\right), \ldots, u\left(t-n_{b}-n_{k}+1\right)\right) .
$$

A major advantage with this structure is that it will always generate bounded predictions (provided, of course, that $m(\cdot)$ does not have any singularities), since it only depends on past inputs which are assumed to be bounded. However, in order to achieve 
accurate predictions, the NFIR structure normally requires quite large model orders $n_{b}$, which, as discussed above, is unsuitable for the local modeling approach.

4.1.2. NARX Structures Augmenting the NFIR model structure with an autoregressive part results in the nonlinear, autoregressive with exogenous input, NARX, model. As shown earlier by Ljung and Wahlberg (1992), the linear FIR and ARX models are capable of approximating any linear system provided arbritrary high model orders are allowed. It easy to believe that the NARX model will play a similar role for the nonlinear case. The most general NARX structure is

$$
\begin{aligned}
& y(t)=m\left(y(t-1), \ldots, y\left(t-n_{a}\right),\right. \\
& \left.u\left(t-n_{k}\right), \ldots, u\left(t-n_{b}-n_{k}+1\right)\right)+e(t),
\end{aligned}
$$

which was referred to as the $\mathrm{NARX}_{1}$ structure by Sjöberg et al. (1995). Here $n_{a}$ denotes the number of lagged outputs. Its predictor is

$$
\begin{aligned}
\hat{y}(t)=m\left(y(t-1), \ldots, y\left(t-n_{a}\right),\right. & \\
& \left.u\left(t-n_{k}\right), \ldots, u\left(t-n_{b}-n_{k}+1\right)\right) .
\end{aligned}
$$

The NARX model has several nice properties which make it suitable to work with and a default choice for many applications:

- It is general and can describe any nonlinear system well.

- It is not recurrent, i.e., the regressors are independent of previous model outputs.

Unfortunately, there are also drawbacks: There is no specific noise model built into the structure. Instead the noise dynamics are modeled along with the plant dynamics. As a consequence of this, a good approximation of both the system and the noise dynamics may require a high dimension of the regression vector $\varphi(t)$. A solution to these problems is to include separate noise dynamics in the model.

4.1.3. Structures with Separate Noise Models: NAR$M A X, N O E$ and NBJ Structures with separate noise models can be constructed by incorporating past prediction errors $\varepsilon(t-k)=y(t-k)-\hat{y}(t-k)$ into the regression vector. However, since it under normal circumstances is quite difficult to model the noise in a general nonlinear way, it seems natural to impose the restriction that the model is linear in the prediction errors. This corresponds to the NARMAX-type structure

$$
y(t)=m(\varphi(t))+C(q) e(t),
$$

where $\varphi(t)$ is constructed as in the NARX case. The corresponding predictor is

$$
\hat{y}(t)=m(\varphi(t))+(C(q)-1) \varepsilon(t),
$$

which can be interpreted as a conditionally parametric model that is global in the $\varepsilon(t-k)$-directions of the regressor space.
A NARMAX model of the form (6) can be estimated by modifying the loss function in (2) according to

$$
\underset{C, \beta}{\arg \min } \sum_{k}\left[C^{-1}(q)(y(k)-m(\varphi(k), \beta))\right]^{2} \cdot w_{k}(\varphi(t))
$$

However, since this model is not linear in the parameters, a numerical optimization routine must be used. Thus this model is not that suitable for the nonparametric setting. The same conclusion will of course also hold for the nonlinear Box-Jenkins (NBJ) and output error (NOE) structures.

4.1.4. NFIR Systems with Colored Noise So far we have assumed that the noise term $e(t)$ is white. Let us see what happens if we remove this assumption. Consider an NFIR model with an additive but not necessarily white noise term $v(t)$,

$$
y(t)=m\left(u(t-1), \ldots, u\left(t-n_{b}\right)\right)+v(t) .
$$

For the noise $v(t)$ we will in general only need a spectral description. It can thus be described by

$$
v(t)=H(q) e(t)
$$

where $\{e(t)\}$ is white noise. A predictor for (7) is

$$
\hat{y}(t)=H^{-1}(q) m\left(\varphi_{u}(t)\right)+\left(1-H^{-1}(q)\right) y(t)
$$

In the first term, the linear filter $H^{-1}(q)$ can equally well be absorbed into the nonlinear mapping $m(\cdot)$. The structure (7) can thus be viewed as a NFIR structure, accomplished by a linear term consisting of past $y(t)$ 's. That is,

$$
\hat{y}(t)=(1-A(q)) y(t)+\bar{m}\left(\varphi_{u}(t)\right) .
$$

Since this structure has the same regressor configuration as the $\mathrm{NARX}_{1}$ model in equation (5), it has been referred to as a NARX $\mathrm{N}_{2}$ model by Sjöberg et al. (1995). The fact that it is not recurrent makes it a very interesting and useful alternative to work with.

In the model-on-demand framework, the $\mathrm{NARX}_{2}$ model can similarly to the NARMAX structure above be interpreted as a conditionally parametric local linear model which is global in the $y(t-k)$-directions of the regressor space. Estimation of such models can be performed by zeroing out the corresponding elements of the scaling matrix $\mathcal{M}$ that controls the distance function. Another alternative is to start by fitting a global linear model to the $y(t-k)$-regressors, and use the residuals for nonparametric modeling in the $u(t-k)$-directions of the regressor space.

The $\mathrm{NARX}_{2}$ concept can be extended to the case where the predictor consists of a sum of two nonlinear terms which are independent of each other. That is

$$
\hat{y}(t)=m_{1}\left(\varphi_{y}(t)\right)+m_{2}\left(\varphi_{u}(t)\right) .
$$

A model of this type is referred to as an additive model in the statistical literature (Hastie and Tibshirani, 1990), and can (although even more computational demanding) be estimated using the backfitting 
algorithm which alternates between estimating $m_{1}(\cdot)$ and $m_{2}(\cdot)$ from previous residuals. In the work by Sjöberg et al. (1995), a model of the form (9) was called a $\mathrm{NARX}_{3}$ model, and can be interpreted as a FIR model of the type (7) where the noise is state dependent. That is,

$$
v(t)=H\left(q, \varphi_{u}(t)\right) e(t) .
$$

\section{AN EXAMPLE}

In this section we will illustrate some of the previously described concepts through simulations. The application has previously been investigated by Sjöberg et al. (1995) and Gunnarsson and Krus (1990) and has to some extent become a "benchmark" example for nonlinear black-box modeling.

The system in question is a hydraulic actuator that controls the position of a robot arm. The arm position is directly coupled to the oil pressure in the actuator which is determined by a valve that regulates the oil flow into the actuator. The modeling aim is to model the oil pressure as a function of the valve opening.

The dataset consists of 1024 samples in total and has been split into two equally sized parts - one for estimation and one for validation. Figure 2 shows the estimation part. The valve opening is here denoted $u(t)$ and the oil pressure $p(t)$. As observed from
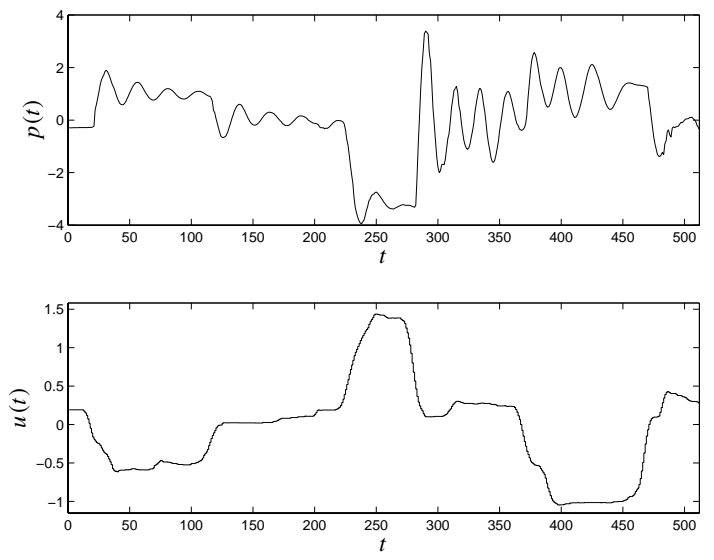

Fig. 2. Measured values of oil pressure $p(t)$ and valve position $u(t)$.

the figure, the oil pressure signal has very oscillative settling periods after step changes in the valve size. This is primarily due to mechanical resonances in the robot arm.

It seems reasonable to start by first considering simple linear black-box models. Experiments performed by Sjöberg et al. (1995) show that a model that predicts the pressure $p(t)$ using three old values of the pressure and two old values of the valve size signal (i.e., an ARX 321 model) is appropriate for this particular application. The result of a simulation on validation data using this configuration is shown in Figure 3 (a). The achieved root mean squared error, RMSE, for the simulated output is 0.925 , but it is quite obvious from visual inspection that it would be possible to improve this rather poor result by considering more complex nonlinear models.

A severe problem with this application from a local modeling viewpoint is of course the sparseness of data (512 data points in a five-dimensional regressor space). However, it might anyway be interesting to see how far we can get with the a local polynomial approach. We found that in order to obtain reasonable results we had to increase the model order by one. That is, to use a NARX 431 predictor,

$$
\begin{array}{r}
\hat{p}(t)=m(p(t-1), p(t-2), p(t-3), p(t-4), \\
u(t-1), u(t-2), u(t-3)) .
\end{array}
$$

Figure 3 (b) shows a simulation on validation data using the model-on-demand concept with a local lin-

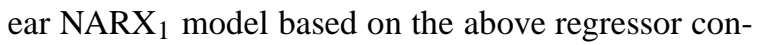
figuration. The achieved RMSE is 0.499 and represents a significant enhancement compared to the linear ARX model. It turns out though that the result can be even further improved by considering a conditionally global NARX $\mathrm{N}_{2}$ model. This can be achieved by altering the scaling matrix according to

$$
\mathcal{M}=\operatorname{diag}\left(\begin{array}{lllllll}
0 & 0 & 0 & 0 & 1 & 1 & 1
\end{array}\right)
$$

A simulation with this setup in the model-on-demand framework is shown in Figure 3 (c). The RMSE error is now 0.471 . Trying the $\mathrm{NARX}_{3}$ model and other choices of model parameters did not result in any further improvements. However, Sjöberg et al. (1995) obtained a best value of 0.328 using a complex neural network structure. The conclusion is therefore that this dataset is too small to produce results directly comparable to the parametric case. Nevertheless, we have demonstrated how local modeling techniques even for rather small datasets quite drastically can improve the quality of the simulations compared to traditional linear black-box modeling.

Table 1. The best achieved RMS errors.

\begin{tabular}{r|l} 
Method & RMSE \\
\hline ARX 321 & 0.926 \\
NARX 431 (MOD) & 0.471 \\
NARX 431 (NN) & 0.328
\end{tabular}

\section{CONCLUSIONS}

We have in this paper discussed several aspects of model structure selection associated with the local polynomial modeling concept, and have shown that the modeling approach is a useful for predicting and simulating nonlinear systems when sufficiently large datasets are available. A number of commonly used model structures have been evaluated from a modelon-demand perspective and we have concluded that the different NARX structures should be considered as the default choice in this context. Furthermore, it has been shown that the predictions in some situations 


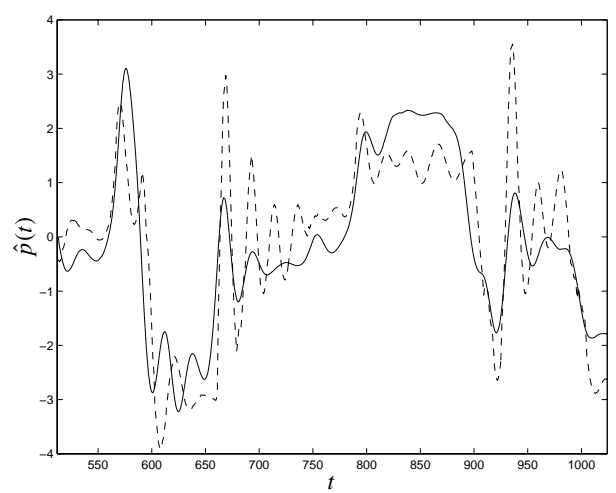

(a)

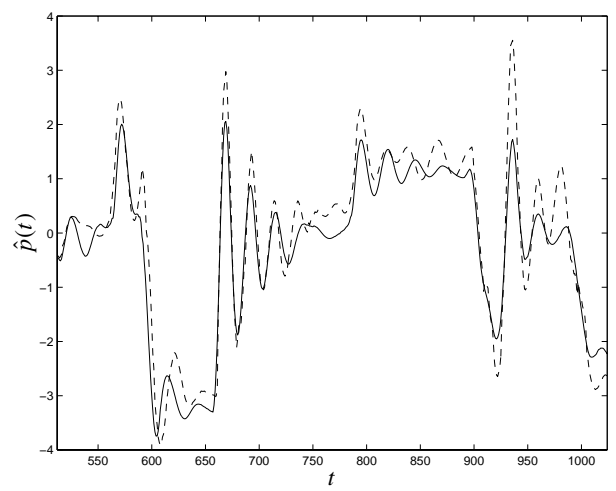

(b)

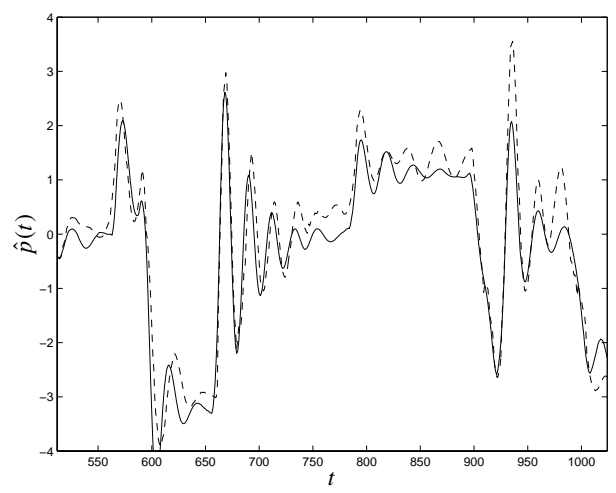

(c)

Fig. 3. Simulation of the crane behavior using validation data: (a) ARX model. (b) NARX 1 model. (c) $\mathrm{NARX}_{2}$ model. The solid lines are the simulated output and the dashed lines are the true system output.

can be enhanced by tuning certain parameters like the the scaling matrix.

The usefulness of the model-on-demand concept has been illustrated in numerical simulations. For the chosen application we have shown that the prediction errors are in order of magnitude directly comparable to more established modeling tools such as artificial neural nets. However, we have at the same time shown that the demand for large datasets is more obvious in the model-on-demand case than for traditional parametric modeling approaches.

\section{REFERENCES}

Bentley, J.L. (1975). Multidimensional binary search trees used for associative searching. Communications of the ACM 18(9), 509-517.

Chen, S., S.A. Billings, C.F.N. Cowan and P.M. Grant (1990). Non-linear system identification using radial basis functions. International Journal of Systems Science 21(12), 2513-2539.

Fan, J. and I. Gijbels (1996). Local Polynomial Modelling and Its Applications. Chapman \& Hall.

Gunnarsson, S. and P. Krus (1990). Modeling of a flexible mechanical system containing hydralic actuators. Technical report. Dept of EE. Linköping University, S-581 83, Linköping, Sweden.

Hastie, T. and R. Tibshirani (1990). Generalized Additive Models. Chapman \& Hall.

Kulhavý, R. and P. Ivanova (1999). Memory-based prediction in control and optimisation. In: Preprints of the 14th World Congress of IFAC (H.F. Chen and B. Wahlberg, Eds.). Vol. H. Elsevier Science. pp. 289-294.

Ljung, L. (1999). System Identification - Theory for the user. 2nd ed.. Prentice-Hall, New Jersey.

Ljung, L. and B. Wahlberg (1992). Asymptotic properties of the least-squares method for estimating transfer functions and disturbance spectra. $A d$ vances in Applied Probability 24, 412-440.

Sjöberg, J., Q. Zhang, L. Ljung, A. Benveniste, B. Deylon, P.-Y. Glorennec, H. Hjalmarsson and A. Juditsky (1995). Nonlinear black-box modeling in system identification: a unified overview. Automatica 31, 1691-1724.

Stenman, A. (1999). Model on Demand: Algorithms, Analysis and Applications. PhD thesis. Dept of EE, Linköping University. SE-581 83, Linköping, Sweden.

Stenman, A., A.V. Nazin and F. Gustafsson (1997). Asymptotic properties of Just-in-Time models. In: Preprints of the 11th IFAC Symposium on System Identification, Kitakyushu, Japan (Y. Sawaragi and S. Sagara, Eds.). pp. 12491254.

Stenman, A., F. Gustafsson and L. Ljung (1996). Just in time models for dynamical systems. In: Proceedings of the 35th IEEE Conference on Decision and Control, Kobe, Japan. pp. 1115-1120.

Tibshirani, J.R. and T.J. Hastie (1987). Local likelihood estimation. Journal of the American Statistical Association 82, 559-567. 